\title{
Segregation of Amphiphilic Polymer-Coated Nanoparticles to Bicontinuous Oil/Water
}

\author{
Microemulsion Phases
}

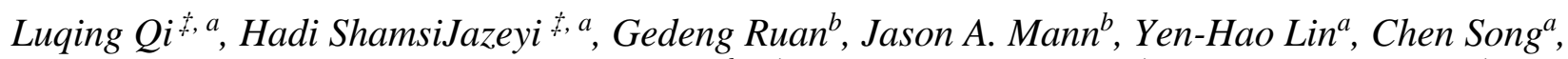
Yichuan Ma ${ }^{a}$, Le Wang ${ }^{a}$, James M. Tour ${ }^{b, c,}$, , George J. Hirasaki ${ }^{a, ~}$, Rafael Verduzco ${ }^{a, c}$, *

${ }^{a}$ Rice University, Department of Chemical and Biomolecular Engineering, 6100 Main Street, MS362, Houston TX, USA, 77005;

${ }^{b}$ Rice University, Department of Chemistry, 6100 Main Street, MS-60, Houston TX, USA, 77005;

'Rice University, Department of Materials Science and NanoEngineering, 6100 Main Street MS325, Houston TX, USA, 77005

Keywords: nanoparticle; polymer; microemulsion; SAXS 


\begin{abstract}
Polymer-coated nanoparticles are interfacially active and have been shown to stabilize macroscopic emulsions of oil and water, also known as Pickering emulsions. However, prior work has not explored the phase behavior of amphiphilic nanoparticles in the presence of bicontinuous microemulsions. Here, we show that properly designed amphiphilic polymer-coated nanoparticles spontaneously and preferentially segregate to the bicontinuous microemulsion phases of oil, water, and surfactant. Mixtures of hydrophilic and hydrophobic chains are covalently grafted onto the surface of oxidized carbon black nanoparticles. By sulfating hydrophilic chains, the polymer-coated nanoparticles are stable in the aqueous phase at salinities up to $15 \mathrm{wt} \% \mathrm{NaCl}$. These amphiphilic, negatively charged polymer-coated nanoparticles segregate to the bicontinuous microemulsion phases. We analyzed the equilibrium phase behavior of the nanoparticles, measured the interfacial tension, and quantified the domain spacing in the presence of nanoparticles. This work shows a novel route to the design of polymer-coated nanoparticles which are stable at high salinities and preferentially segregate to bicontinuous microemulsion phases.
\end{abstract}




\section{INTRODUCTION}

The demand for oil is expected to continue to increase over the next several decades, ${ }^{1}$ and methods to improve oil recovery and production are needed. Primary and secondary oil recovery methods can only produce $15-30 \%$ of the original oil-in-place through water flooding. Tertiary recovery methods, also known as enhanced oil recovery (EOR), can lead to further production, up to $65 \%$ of the original oil-in-place depending on the approach. ${ }^{2,3} \mathrm{~A}$ variety of chemicals and additives are used in EOR processes, including surfactants, emulsifiers, alkali, and polymers, among other additives. ${ }^{4}$

Nanoparticles are of interest as additives for EOR processes since they can be used to tailor interfaces and can propagate through porous media. ${ }^{5}$ A number of studies have focused on micronand nano-sized surface active nanoparticle additives for stabilizing oil-water emulsions, mobility control of the injected flood, and wettability alteration of the surface to increase the efficiency of EOR processes. For example, Binks et al. studied water-in-oil macro-emulsions stabilized by Latex particles and found that, in the case of micron-size colloidal particles, the interaction with the interface was dominated by irreversible adsorption. ${ }^{6,7}$ The group also demonstrated that silica nanoparticles could be used to stabilize oil-in water macro-emulsions when blended with anionic or cationic surfactant, respectively. ${ }^{8,9}$ Johnston and coworkers studied a series of iron-oxide nanoparticles coated with amphiphilic ${ }^{10}$ or charged polymers ${ }^{11,12}$ and found a decrease in the value of oil-water interfacial tension and, in the case of charged polymer coatings, both good stability in high-salinity environments and low adsorption onto silica surfaces. Johnston, Krishnamoorti, and coworkers also studied iron-oxide clusters and silica nanoparticles coated with poly[oligo(ethylene oxide) monomethyl ether methacrylate] and demonstrated a significant reduction in interfacial tension at very low nanoparticle concentrations $(1-10 \mathrm{ppm}) \cdot{ }^{13,14}$ Harwell and coworkers 
demonstrated that surfactants could be used to disperse multi-walled carbon nanotubes in aqueous solution and transport them through porous media with little or no adsorption. ${ }^{15,16}$ Cui et al. found that nanoparticle/surfactant mixtures could be used to deform emulsion droplets into non-spherical shapes due to jamming of the nanoparticles at the oil-water interface. ${ }^{17}$ Lead and coworkers reported polymer-coated nanoparticles that could be used to separate oil-water mixtures. ${ }^{18,19}$ Other studies with polymer-coated nanoparticles at the oil-water interface are discussed in recent review articles. $^{20-22}$

Bicontinuous microemulsions are thermodynamically stable phases formed by a mixture of oil, water and surfactant. ${ }^{23-25}$ In addition to bicontinuous, nanoscopic domains of oil and water, these phases are characterized by an ultralow interfacial tension between oil and water. As such, these phases are of interest for surfactant-enhanced oil recovery, in which oil can be solubilized and mobilized in the bicontinuous microemulsion phase. ${ }^{4,26}$ While prior work has clearly demonstrated the potential of polymer-coated nanoparticles for stabilizing emulsions, previous studies have not investigated the interaction of such nanoparticles with oil-water microemulsions.

Here, we propose that properly designed, polymer-coated nanoparticles can segregate to surfactant-stabilized oil-water microemulsions, as shown schematically in Figure 1. We prepare a series of oxidized carbon black (OCB) nanoparticles with a mixture of hydrophilic and hydrophobic chains covalently grafted to the surface. We find that nanoparticles coated with a mixture of negatively charged polymer chains and hydrophobic chains are stable in water at elevated salinities (up to $15 \mathrm{wt} \% \mathrm{NaCl}$ ). When mixed with surfactants that stabilize the formation of bicontinuous oilwater phases, the polymer-coated nanoparticles migrate preferentially to the bicontinuous microemulsion phase. We detail the preparation of the polymer-coated nanoparticles, their phase behavior in oil-water-surfactant mixtures, and their impact on oil-water interfacial properties. This 
work points to a novel property of polymer-coated nanoparticles and their specific interaction with bicontinuous phases. Combined with other favorable properties of nanoparticle additives, we envision this work will lead to future developments of nanoparticle additives which can segregate to bicontinuous phases, propagate through porous media, modify interfacial properties, and enhanced the efficiency of enhanced oil recovery processes.

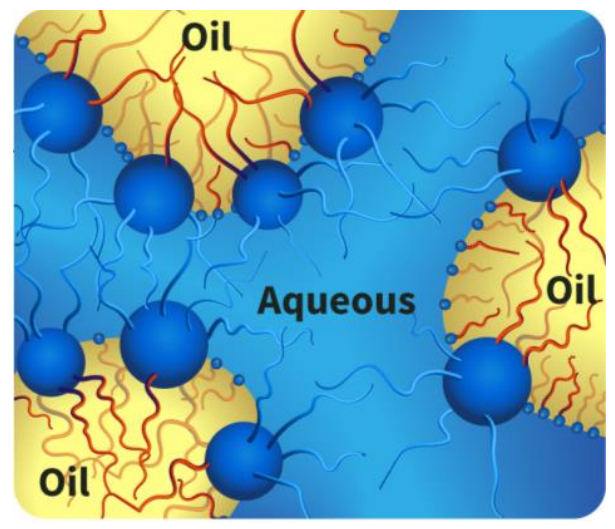

Figure 1. Schematic of oil-water bicontinuous microemulsion phases with polymer-coated nanoparticle additives.

\section{EXPERIMENTAL}

Materials. Carbon black nanoparticles were purchased from Cabot Corporation (Monarch 1300, Lot \# 944884), with a diameter of $15 \mathrm{~nm}$. Solvents and reagents were obtained from commercial suppliers and used as received. $\mathrm{C}_{12}$-orthoxylene sulfonate (OXS) surfactant was provided by Exxon, S13B 23-71SM surfactant was provided by TIORCO, and ALFOTERRA series surfactants (AF K2-4 1S and AF K3-4 1S) were obtained from Sasol. The surfactants used in this study are shown in Figure 2. 


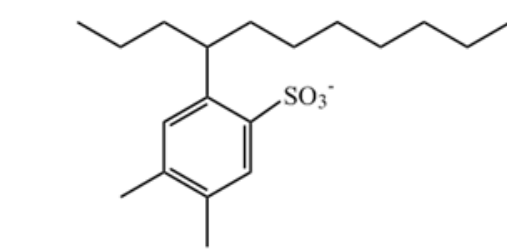

(a)

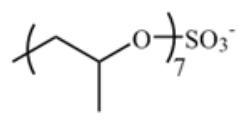

(b)

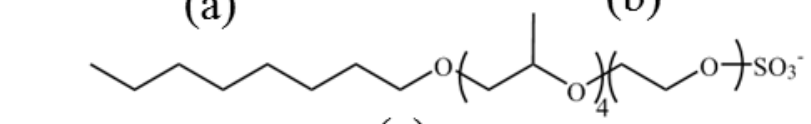

(c)

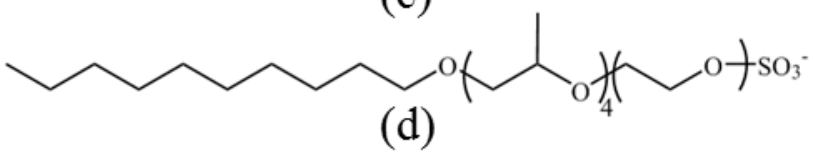

(d)

Figure 2. Chemical structures for surfactants used in this study. (a) OXS (b) S13B 23-71SM (C) ALFOTERRA K2-4 1S (d) ALFOTERRA K3-4 1S

Oxidation of OCB. Oxidized carbon black nanoparticles (OCB) were prepared as follows. 9:1 v/v mixture of concentrated $\mathrm{H}_{2} \mathrm{SO}_{4} / \mathrm{H}_{3} \mathrm{PO}_{4}(360: 40 \mathrm{~mL})$ was added to a mixture of carbon black nanoparticles $(3.0 \mathrm{~g})$ and $\mathrm{KMnO}_{4}(18.0 \mathrm{~g})$. The reaction was heated to $50{ }^{\circ} \mathrm{C}$ and stirred for $12 \mathrm{~h}$. The reaction was then cooled to room temperature and poured over ice $(\sim 400 \mathrm{~mL})$ with $30 \% \mathrm{H}_{2} \mathrm{O}_{2}$ ( $3 \mathrm{~mL})$. The dispersion was then centrifuged $(6000 \mathrm{rpm}, 4 \mathrm{~h})$ and the supernatant discarded. The remaining solid material was then washed with $200 \mathrm{~mL}$ of $30 \% \mathrm{HCl}$ two times, each time followed by centrifugation and decanting of the supernatant. Finally, the OCB nanoparticles were dialyzed in water for one week using dialysis membrane (MWCO of $1 \mathrm{kD})$. The resulting nanoparticles were then aggregated by adding hexanes and then collected by filtration and dried under vacuum.

PVA-OCB. Hydrophilic poly(vinyl alcohol) (PVA)-coated OCB nanoparticles were prepared by covalently attaching PVA to the OCB surface. First, OCB (25 mg) and PVA (2000 g/mol, 75-80 \% hydrolyzed, $2.1 \mathrm{~g}$ ) were dispersed in DMF (25 mL). Next, $N, N^{\prime}$-dicyclohexylcarbodiimide (DCC, $206 \mathrm{mg}$ ) and 4-(dimethylamino)pyridine (DMAP, 100 mg) were added to the reaction. The reaction mixture was bath ultrasonicated for 10 min prior to heating to $70{ }^{\circ} \mathrm{C}$ for one day. The resulting polymer-coated nanoparticles were dialyzed in water for one week using dialysis 
membrane (MWCO of $50 \mathrm{kD}$ ). The nanoparticles were aggregated by adding hexanes and then collected by filtration and dried under vacuum.

C16-PVA-OCB. Amphiphilic OCB nanoparticles were prepared by covalently attaching PVA and hydrophobic $\mathrm{C}_{16} \mathrm{H}_{33} \mathrm{OH}$ (C16) chains to the surface. First, OCB (25 mg), DCC (206 mg), DMAP (100 mg), and C16 (250 mg) were dispersed in DMF (25 mL). The reaction was carried out at 90 ${ }^{\circ} \mathrm{C}$ for 3 days. Next, PVA was attached to the surface of these nanoparticles. PVA ( $\left.2.1 \mathrm{~g}\right)$ was added to the reaction along with additional DCC $(150 \mathrm{mg})$, the reaction mixture was bath ultrasonicated for $10 \mathrm{~min}$ prior to stirring at $70{ }^{\circ} \mathrm{C}$ for one day. The resulting polymer-coated nanoparticles were dialyzed in DMF for 3 days at $50{ }^{\circ} \mathrm{C}$ and then in water for one week using dialysis membrane (MWCO of $50 \mathrm{kD}$ ). The nanoparticles were aggregated by adding hexanes and then collected by filtration and dried under vacuum.

sPVA-OCB, C16-SPVA-OCB and C16-ssPVA-OCB. Polymer-coated nanoparticles with sulfated PVA (sPVA) chains attached were prepared by sulfating PVA-OCB or $\mathrm{C}_{16}$-PVA-OCB nanoparticles. For conducting full sulfation, sulfur trioxide pyridine complex $(26 \mathrm{mg})$ was added to the nanoparticle $(32 \mathrm{mg})$ dispersion in DMF $(1.5 \mathrm{~mL})$ at $60{ }^{\circ} \mathrm{C}$ under nitrogen purge, and the reaction was allowed to proceed for 1 day. For conducting partial sulfation, $6.4 \mathrm{mg}$ of the pyridine complex is added to the same amount of nanoparticle (32 mg). sPVA-OCB was dialyzed in water for one week using dialysis membrane (MWCO of $50 \mathrm{kD}$ ). $\mathrm{C}_{16}$-sPVA-OCB and $\mathrm{C}_{16}$-ssPVA-OCB were dialyzed in DMF for 3 days at $50^{\circ} \mathrm{C}$ and then in water for one week using dialysis membrane (MWCO of $50 \mathrm{kD}$ ). The nanoparticles were aggregated by adding hexanes and then collected by filtration and dried under vacuum. 


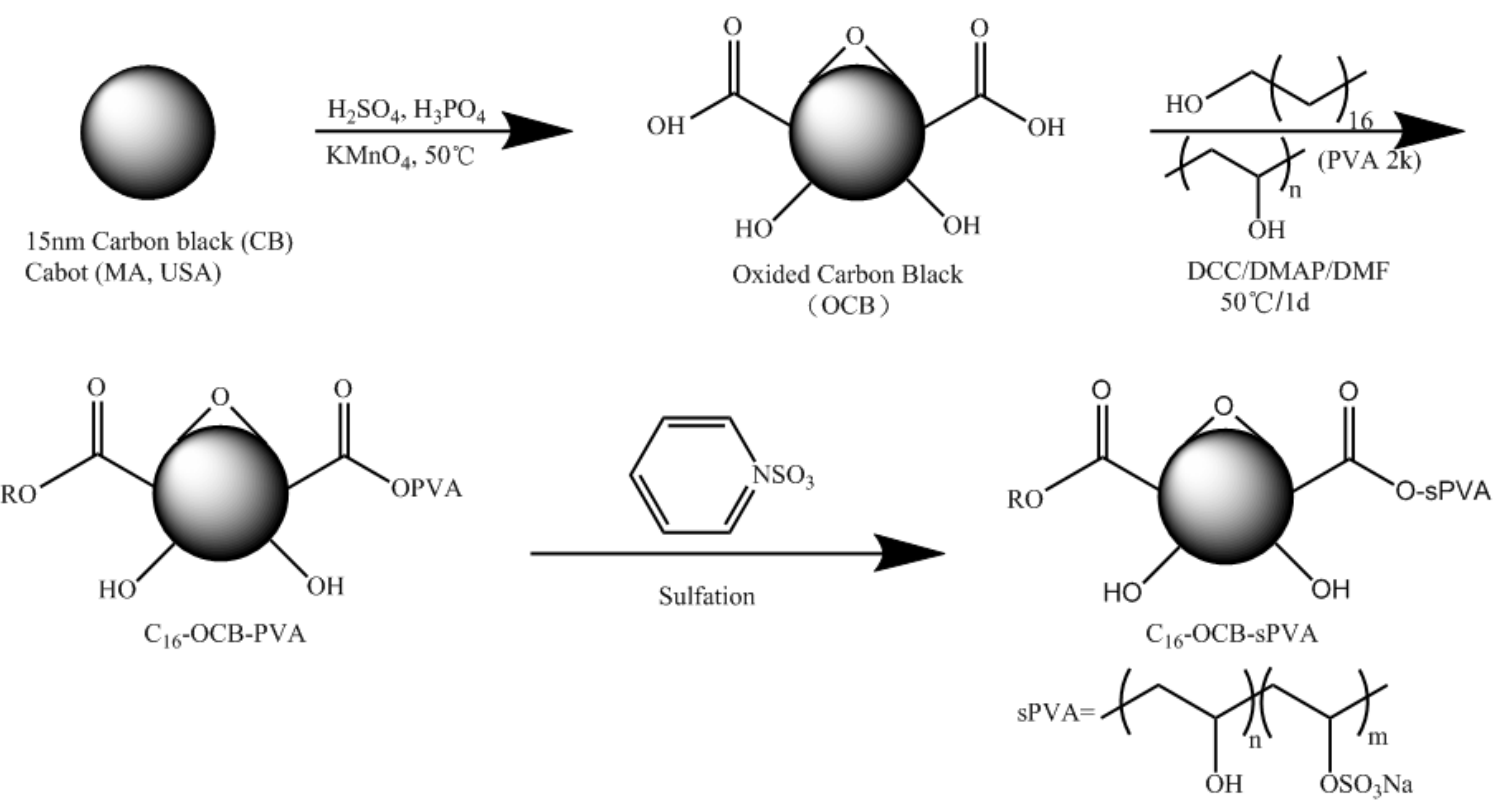

Figure 3. Reaction scheme for the surface modification of oxidized carbon black nanoparticles (OCB). sPVA: lightly sulfated PVA, ssPVA: highly sulfated PVA

Dynamic Light Scattering (DLS) Measurement. Dynamic light scattering was carried out using a Malvern Instruments Zen 3600 Zetasizer. Polymer-coated nanoparticles were dispersed in water at a concentration of 5-10 ppm for analysis.

Phase Behavior Studies. Phase behavior studies of oil, water, surfactant, and polymer-coated nanoparticle were carried out using $3 \mathrm{~mL}$ made from cutting $5 \mathrm{~mL}$ borosilicate, serological pipettes. First, the pipettes were filled with the desired amount of brine (aq. $\mathrm{NaCl}$ ), oil ( $n$-octane), surfactant, and polymer-coated nanoparticle. Except where noted, the surfactant was 2 vol $\%$ of the aqueous phase and the polymer-coated nanoparticle concentration was $0.2 \mathrm{wt} \%$ of the aqueous phase. 2.5 wt \% sec-butyl alcohol was added to avoid the formation of thick macro-emulsion phases. To equilibrate the mixture in each pipette, pipettes were sealed with a torch prior to mixing and mixed overnight on a shaker. The final mixtures were allowed to rest at room temperature and photographed when the phase was stabilized. 
Thermo-Gravimetric Analysis (TGA). TGA measurements were carried out on a Q-600 TGA from TA instruments. The samples were heated from $25^{\circ} \mathrm{C}$ to $900{ }^{\circ} \mathrm{C}$ at a rate of $10{ }^{\circ} \mathrm{C} / \mathrm{min}$ under nitrogen atmosphere.

Interfacial Tension (IFT) Measurement. A spinning drop interfacial tensiometer (Krüss SITE100) was used to measure oil-water IFT.

Small-angle X-ray Scattering. Small angle X-ray scattering (SAXS) measurements were carried out on Sector 8-ID-E at the Advanced Photon Source Argonne National Laboratory. Beamline 8ID-E operates at energy of $7.35 \mathrm{keV}$, and images were collected from a Pilatus 1MF camera (Dectris), with two exposures for different vertical position of the detector. After flat-field correction for detector nonuniformity, the images were combined to fill in the gaps for rows at the borders between modules, leaving dark only the columns of inactive pixels at the center. Using the GIXSGUI package for MATLAB (MathWorks), ${ }^{27}$ data were corrected for X-ray polarization, detector sensitivity, and geometrical solid angle.

SAXS samples were prepared from equilibrated phase behavior samples. Extracts were carefully taken from the microemulsion layer of phase-separated samples and transferred to quartz capillaries (Hampton Research, $\mathrm{OD}=1.5 \mathrm{~mm}$ ). Immediately after that the top of capillary was sealed with wax. A series of ten samples were aligned onto a specially-made holder and then transferred into a vacuum chamber. Sample measurements were carried out under vacuum which is in the range of $2 \sim 3 \times 10^{-6}$ bar, with the sample stage interfaced with a Lakeshore 340 unit. The beam size is $200 \mu \mathrm{m}$ (h) $\mathrm{x} 20 \mu \mathrm{m}$ (v). Sample detector distance was $208 \mathrm{~mm}$. 


\section{RESULTS AND DISCUSSION}

A series of polymer-coated nanoparticles were prepared by either attaching hydrophilic poly(vinyl alcohol) (PVA) chains or a mixture of hydrophilic PVA and hydrophobic $\mathrm{C}_{16}$ alkyl chains to the surface of oxidized carbon black (OCB), as shown in Figure 3. Surface functionalities on carbon black nanoparticles were introduced through oxidation, and hydrophobic or hydrophilic chains were covalently grafted to the nanoparticle surface through a Steglich esterification reaction between carboxylate groups on the nanoparticle surface and hydroxyl groups on the polymer. In order to improve nanoparticle stability in high-salinity aqueous media, selected polymer-coated nanoparticles were partially sulfated, resulting in nanoparticles coated with charged sulfated PVA (sPVA) chains. The phase behavior of these nanoparticles in aqueous media is detailed below.

The polymer content and size of the nanoparticles was measured through thermogravimetric analysis (TGA) and dynamic light scattering (DLS), respectively, and the results are shown in Table 1 and Supporting Information Figure S1. The polymer content is greater than $70 \mathrm{wt}$ $\%$ for all samples prepared, and an increase in the size of the polymer-coated nanoparticles is observed after sulfation, which we attribute to electrostatic interactions between the charged sPVA chains on the nanoparticle surface. The lower polymer content for $\mathrm{C}_{16}$-ssPVA-OCB may be due to partial hydrolysis and removal of the chains attached to the nanoparticle surface during the extended sulfation reaction. This is also reflected in a drop in size of the $\mathrm{C}_{16}$-ssPVA-OCB nanoparticles after extended sulfation relative to $\mathrm{C}_{16}$-sPVA-OCB. 
Table.1 Polymer fraction by TGA and size measurement by DLS

\begin{tabular}{ccc} 
Nanoparticle & $\begin{array}{c}\text { Polymer content } \\
(\text { wt } \%)\end{array}$ & $\begin{array}{c}\text { Size }^{\mathrm{b}} \\
(\mathrm{nm})\end{array}$ \\
\hline OCB & 0 & 50 \\
\hline PVA-OCB & 91 & 54 \\
sPVA-OCB & 81 & 170 \\
C $_{16}$-PVA-OCB & 91 & 72 \\
$\mathrm{C}_{16}$-sPVA-OCB & 97 & 151 \\
$\mathrm{C}_{16}$-ssPVA-OCB & 72 & 123 \\
\hline
\end{tabular}

${ }^{a}$ measured by thermogravimetric analysis. ${ }^{b}$ measured by dynamic light scattering in DI water. OCB: oxidized carbon black; PVA: poly(vinyl alcohol); sPVA: lightly-sulfated PVA; ssPVA: highly-sulfated PVA.

We investigated the equilibrium phase behavior of polymer-coated nanoparticles in the presence of oil and water of varying salinity. The nanoparticle concentration was kept at $0.2 \mathrm{wt} \%$, and the dispersion of the nanoparticle was monitored as a function of changing water salinities. Mixtures were equilibrated by shaking overnight at ambient temperature and then allowing 2 days for oil and aqueous phases to separate. We used a paraffinic, transparent oil (n-octane), and the distribution of nanoparticles can be identified visually, as shown in Figure 4. The polymeric coating had a significant impact on the stability of the nanoparticle dispersion. Unmodified OCB and PVAOCB were only dispersed in the aqueous phase at low salinities but were aggregated at higher salinities (Figure 4a and b). These nanoparticles do not stabilize oil-water emulsions and aggregate at elevated salinities, (0-4 wt \% $\mathrm{NaCl}$ for OCB and $4-8 \mathrm{wt} \% \mathrm{NaCl}$ for PVA-OCB).

By contrast, polymer-coated nanoparticles with a charged polymer coating (sPVA-OCB and $\mathrm{C}_{16}$-sPVA-OCB) remained dispersed in the aqueous phase over a range of salinities. As shown in Figures $4 \mathrm{c}$ and $\mathrm{d}$, these polymer-coated nanoparticles do not aggregate even at salinities as high as 16 wt $\% \mathrm{NaCl}$. The particles were surface active and stabilized the formation of Pickering macroemulsion phases, which did not break even after weeks at room temperature. The amphiphilic 
nanoparticles $\mathrm{C}_{16}$-sPVA-OCB were found to stabilize Pickering macro-emulsions over a much broader range of salinities, from 0 to 16 wt $\% \mathrm{NaCl}$, while the more hydrophilic sPVA-OCB nanoparticles stabilized macro-emulsions over a narrower salinity range of 5.3-11.4 wt \% $\mathrm{NaCl}$ (Fig. $4 c)$.

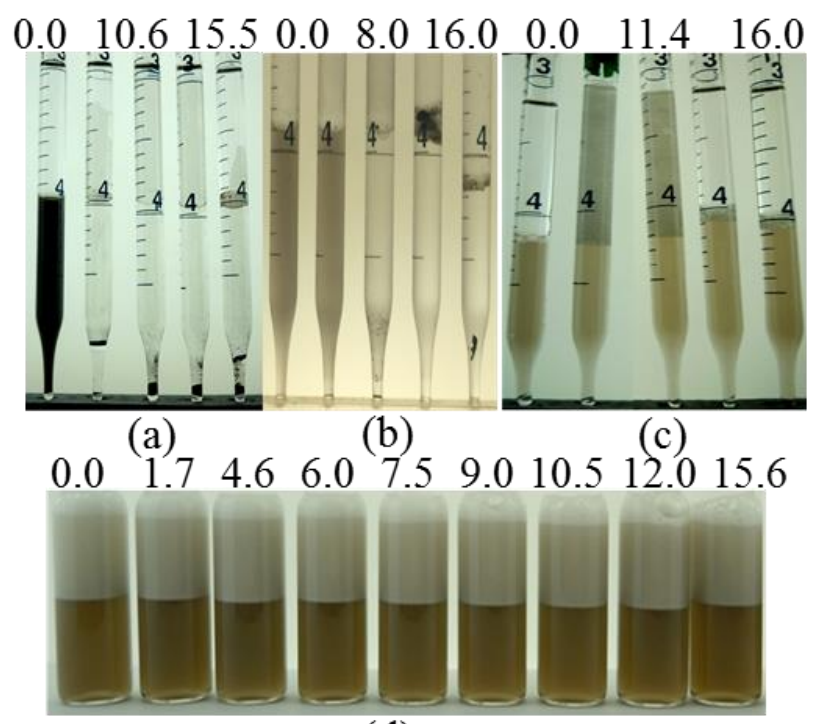

(d)

Figure 4. Equilibrium phase behavior for nanoparticles $(0.2 \mathrm{wt} \%)$ in oil and water: (a) uncoated OCB; (b) PVA-OCB nanoparticles; (c) sPVA-OCB nanoparticles; (d) $\mathrm{C}_{16}$-sPVA-OCB nanoparticles. The upper phase is the oil phase and the bottom phase the aqueous phase for all samples shown.

While the polymer-coated nanoparticles do not produce ultralow oil-water interfacial tensions $\left(<10^{-2} \mathrm{mN} / \mathrm{m}\right)$ and bicontinuous phases, polymer-coated nanoparticles do exhibit surface activity. As shown in the Supporting Information Figure S2, a dispersion of $\mathrm{C}_{16}$-ssPVA-OCB nanoparticles exhibit a $25 \mathrm{mN} / \mathrm{m}$ decrease in surface tension (from 70 to $45 \mathrm{mN} / \mathrm{m}$ ) at a concentration of just $500 \mathrm{ppm}(0.05 \mathrm{wt} \%)$. Furthermore, a measurement of the interfacial tension at the oil-water interface shows a decrease from approximately $50 \mathrm{mM} / \mathrm{m}$ to $17.1 \mathrm{mN} / \mathrm{m}$ (sPVA-OCB) and $11.6 \mathrm{mN} / \mathrm{m}\left(\mathrm{C}_{16}\right.$-ssPVA-OCB$)$ at just $0.1 \mathrm{wt} \%$ polymer-coated nanoparticles, as shown in the Supporting Information Table S1. These reductions in oil-water interfacial tension are comparable to other nanoparticles in the literature, for example silica nanoparticles coated with 
poly(oligo(ethylene oxide) monomethyl ether methacrylate) which exhibit a reduction down to 10 $\mathrm{mN} / \mathrm{m}$ for $<10 \mathrm{ppm}$ nanoparticles. ${ }^{13}$ These interfacial tension values are still too high, however, to stabilize a bicontinuous oil-water microemulsion phase.

To investigate the interaction of polymer-coated nanoparticles with such bicontinuous microemulsion phases, surfactants were added to the oil-water-nanoparticle mixture. Prior studies have been looked at mixtures of nanoparticles and surfactants in solution, ${ }^{28-31}$ but not for the formation of bicontinuous oil-water phases. Four different surfactants were studied, as shown in Figure 2, each of which stabilizes a bicontinuous oil-water phase over a characteristic salinity range. Over this salinity range, the oil-water interfacial tension is ultralow $\left(<10^{-2} \mathrm{mN} / \mathrm{m}\right)$, and the optimal salinity is denoted as the minimum in oil-water interfacial tension. ${ }^{32}$ Figure 5 shows an example phase behavior study of OXS surfactant without nanoparticle (Fig. 5a) and with either $\mathrm{C}_{16}$-sPVAOCB polymer-coated nanoparticle (Fig. 5b) or $\mathrm{C}_{16}$-ssPVA-OCB polymer-coated nanoparticle (Fig. 5c). The bicontinuous microemulsion phase can be visually distinguished as a hazy 'middle phase' between the upper oil phase and lower aqueous phase. OXS surfactant stabilized the formation of a bicontinuous microemulsion phase over the salinity range of approximately $1.6-1.9 \mathrm{wt} \% \mathrm{NaCl}$. At salinities below $1.6 \mathrm{wt} \% \mathrm{NaCl}$ an oil-in-water macro-emulsion (lower phase) is observed and at salinities above 1.9 wt $\% \mathrm{NaCl}$ a water-in-oil macro-emulsion (upper phase) is observed.

With the addition of $\mathrm{C}_{16}$-sPVA-OCB and $\mathrm{C}_{16}$-ssPVA-OCB polymer-coated nanoparticles, the middle phase persists and, notably, nanoparticles segregated exclusively to bicontinuous microemulsion phases. As shown in Figures $5 \mathrm{~b}$ and $\mathrm{c}$, the nanoparticles were clearly observed inhabiting only the middle phase over the range of salinities where a middle phase microemulsion phase forms. No nanoparticles were present in the upper oil or lower aqueous phases when a middle phase is present. At lower and higher extremes in salinity, the nanoparticles segregated to oil-in- 
water macro-emulsion or water-in-oil macro emulsion phases, respectively. In the presence of the polymer-coated nanoparticles, bicontinuous microemulsion phases were formed over a similar range of salinities, with a slight shift to higher salinities on the addition of polymer-coated nanoparticles.

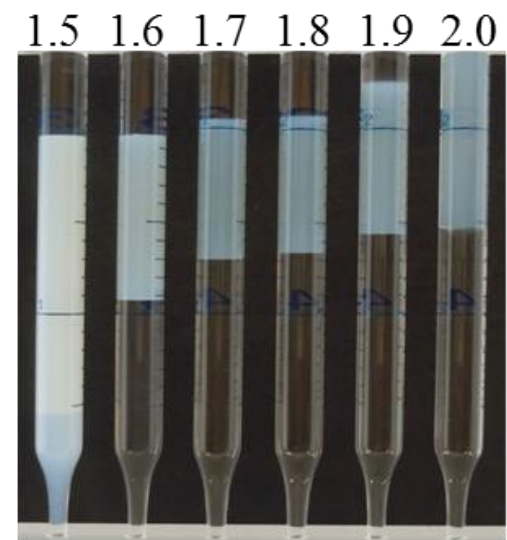

(a)

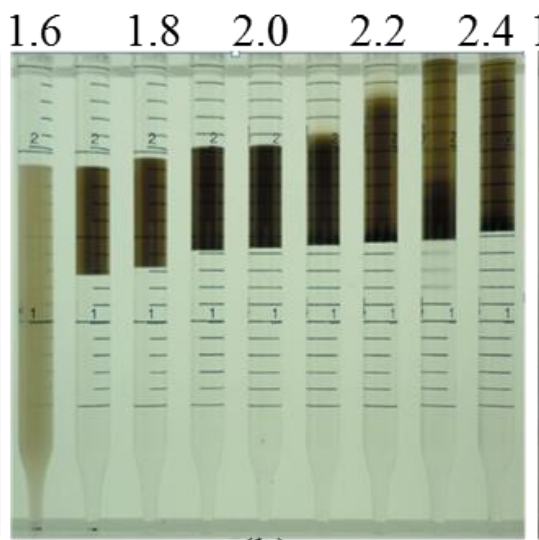

(b)

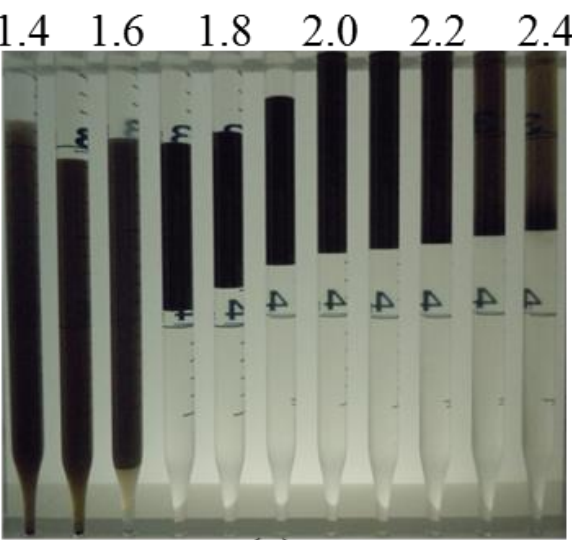

(c)

Figure 5. Phase behavior of $\mathrm{C}_{12}$-orthoxylene sulfonate (OXS) and polymer-coated nanoparticles in octane-water mixtures: (a) OXS (2 wt \%) (b) OXS (2 wt \%) and 0.1wt \% $\mathrm{C}_{16}$-sPVA-OCB and (c) OXS (2 wt \%) and $0.1 \mathrm{wt} \% \mathrm{C}_{16}$-ssPVA-OCB. $2.5 \mathrm{wt} \%$ sec-butyl alcohol was added to all samples shown.

While both charged amphiphilic polymer-coated nanoparticles $\left(\mathrm{C}_{16-\mathrm{SPVA}} \mathrm{OCB}\right.$ and $\mathrm{C}_{16^{-}}$ ssPVA-OCB) segregated exclusively to bicontinuous microemulsion phases of surfactants with relatively low optimal salinities, the degree of sulfation affected the stability of dispersed nanoparticles in the aqueous media. For example, in the cases of S13B surfactant which exhibits an optimal salinity near 4.3 wt $\%$, we observed aggregation of $\mathrm{C}_{16}$-sPVA-OCB nanoparticles. By comparison, the more highly sulfated $\mathrm{C}_{16}$-ssPVA-OCB nanoparticles were stable at these higher salinities and segregated to the bicontinuous middle phases, as shown in the Supporting Information Figure S3

Next, we tested the phase behavior of the highly sulfated $\mathrm{C}_{16}$-ssPVA-OCB nanoparticles in the 
presence of surfactants with ultra-high optimal salinities, i.e. K3-4 $1 \mathrm{~S}$ and K2-4 1S. These surfactants exhibit optimal salinities at approximately $12 \mathrm{wt} \% \mathrm{NaCl}$ and $15 \mathrm{wt} \% \mathrm{NaCl}$, as shown in Figures 6 a and c. Even at these extreme salinities, $\mathrm{C}_{16}$-ssPVA-OCB remained dispersed and segregated exclusively to the oil-water middle phase. Aggregation of the nanoparticles was observed at 16 wt $\% \mathrm{NaCl}$, as shown in Fig. $6 \mathrm{~d}$.

These results are surprising since most nanoparticles are not stable at such high salinities and the amphiphilic nanoparticles were found to segregate strongly and exclusively to bicontinuous microemulsion phases. Such segregation of nanoparticles to microemulsion middle phases has not been previously reported. These phase behavior studies clearly demonstrate that the use of an amphiphilic nanoparticle coating along with a charged, highly sulfated polymer results in polymercoated nanoparticles that are stable at high salinities and migrate to microemulsion middle phases.

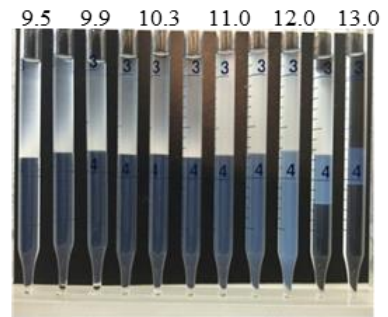

(a)

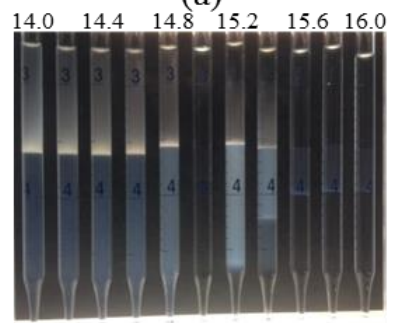

(c)

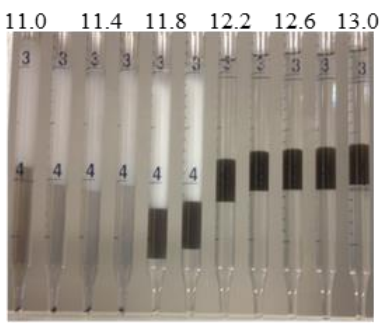

(b)

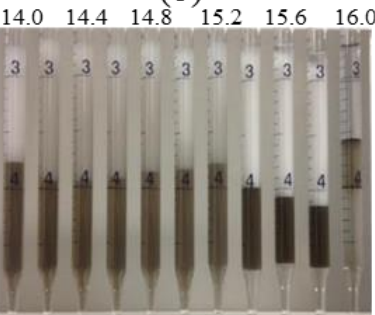

(d)

Figure 6. Phase behavior of K 3-4 1S, K 2-4 1S, and polymer-coated nanoparticle additives: (a) 2 wt \% K3-4 1S (b) 2 wt \% K3-4 $1 \mathrm{~S}$ and 0.1 wt \% C 16 -ssPVA-OCB (c) 2 wt $\% \mathrm{~K} 2-41 \mathrm{~S}$ (d) 2 wt $\% \mathrm{~K} 2-41 \mathrm{~S}$ and $0.1 \mathrm{wt} \% \mathrm{C}_{16}$-ssPVA-OCB. $2.5 \mathrm{wt} \%$ sec-butyl alcohol is added in all cases. 


\section{Influence of Polymer-coated Nanoparticles on Interfacial Tension (IFT) of Oil-Water-}

Surfactant Microemulsion. The formation of bicontinuous microemulsion phases reflect an ultralow oil-water interfacial tension (IFT), and we were interested in understanding the impact of the polymer-coated nanoparticles on the interfacial tension and domain size. Two methods were used to measure the oil-water IFT. First, the interfacial tension was directly measured through spinning drop tensiometry. A second approach was the application of an empirical relationship for the IFT for microemulsion phases that can be extracted from the phase behavior studies, as reported by Huh. ${ }^{33}$ This approach involves measuring the volume of oil $\left(V_{O}\right)$ and water $\left(V_{w}\right)$ incorporated into the middle phase along with the total volume of surfactant $\left(V_{s}\right)$. These can be determined through the equilibrium phase behavior studies described above by determination of the relative volumes of the upper, lower, and middle phase. The IFT can then be given by:

$$
\sigma_{m o}=C /\left(V_{O} / V_{S}\right)^{2}, \quad \sigma_{m w}=C /\left(V_{W} / V_{S}\right)^{2},
$$

where $\sigma_{m o}$ is IFT between the microemulsion phase and the oil phase, $\sigma_{m w}$ the IFT between the microemulsion phase and the water phase, and $C$ is a constant equal to $0.3 \mathrm{mN} / \mathrm{m} .{ }^{33}$ The ratios $V_{o} / V_{s}$ and $V_{w} / V_{s}$ are known as the solubilization parameters for oil and water, respectively, and they reflect the amount of oil or water present in the microemulsion phases normalized by the volume of surfactant $V_{S}$. For these studies, we only focused on the OXS surfactant series, since this particular series provided very clear boundaries between oil, water, and bicontinuous phases, enabling measurement and analysis through the empirical correlation.

Direct measurement of the oil-water interfacial tension through spinning drop IFT indicated that the nanoparticle additive had only a small impact on the IFT. We measured values of $1.8 \times 10^{-3}$ and $1.5 \times 10^{-3} \mathrm{mN} / \mathrm{m}$ with and without nanoparticle added, respectively (with $2 \mathrm{wt} \%$ OXS surfactant and 2.5 wt $\%$ sec-butyl alcohol). In Figure 7, we show the solubilization parameters for water and 
oil in microemulsion phases stabilized by OXS surfactant and both with and without $\mathrm{C}_{16}$-ssPVAOCB polymer-coated nanoparticle. At low salinities, an oil-in-water macro-emulsion (lower phase) is observed, as reflected by a much larger solubilization parameter for water. At higher salinities, a water-in-oil macro-emulsion (upper phase) is present, and solubilization parameter for oil is higher than that for water. Over the salinity range of $1.6-2.0 \mathrm{wt} \% \mathrm{NaCl}$, the solubilization parameters for oil and water are comparable, and this corresponds to the formation of a middle phase. The minimum in interfacial tension (optimal salinity) occurs at the point where the solubilization parameters cross. ${ }^{34}$ These phase behavior studies clearly show that the solubilization parameters are comparable both with and without nanoparticle present and that the optimal salinity occurs at approximately $1.8 \mathrm{wt} \% \mathrm{NaCl}$. Using the empirical Huh correlation, ${ }^{33}$ the IFT is 2.6 and $2.7 \times 10^{-3}$ $\mathrm{mN} / \mathrm{m}$ with and without polymer-coated nanoparticle added, respectively. Thus, both IFT measurement methods indicate that nanoparticle additives have only a small impact on the IFT.

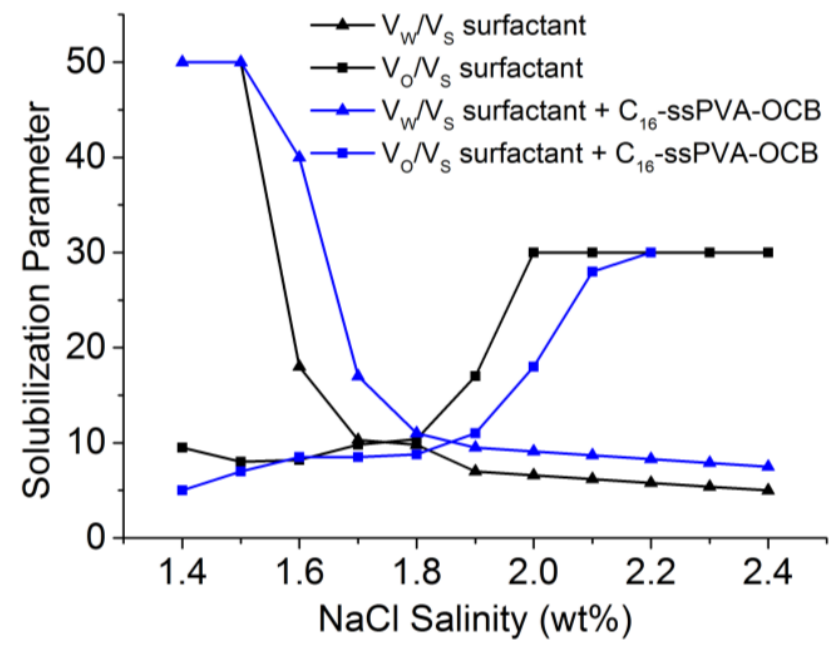

Figure 7. The solubilization parameters for octane-water-OXS mixtures both with and without $\mathrm{C}_{16^{-}}$ ssPVA-OCB (0.1 wt \%) nanoparticle added. 2 wt \% OXS and 2.5 wt \% sec-butyl alcohol were used in all cases. 


\section{Influence of Polymer-coated Nanoparticles on Domain Size of Oil-Water-Surfactant}

Microemulsion. Next, we investigated the effect that polymer-coated nanoparticles had on the domain size of bicontinuous middle phases through small-angle X-ray scattering measurements. In order to carry out these measurements, a sample of the bicontinuous middle phase was extracted from equilibrated oil/water/surfactant/nanoparticle samples and transferred to a capillary for analysis. Microemulsion phases for OXS, K2-4, and K3-4 surfactant series were studied, but only the K2-4 and K3-4 samples provided a measureable correlation peak over the $q$-range of the X-ray measurements, as shown in Figure 8 (data for OXS surfactant samples are shown in the Supporting Information Figure S4). A clear low-angle peak characteristic of a bicontinuous oil-water microemulsion phase was observed in these samples. In both cases, a small shift to lower- $q$ values was seen on the addition of the $\mathrm{C}_{16}$-ssPVA-OCB polymer-coated nanoparticles. This indicated an increase in domain spacing with the addition of polymer-coated nanoparticles.

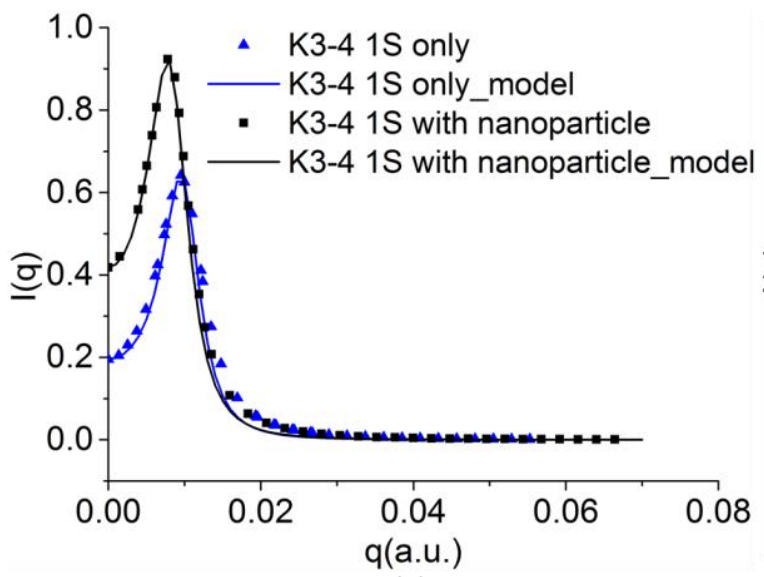

(a)

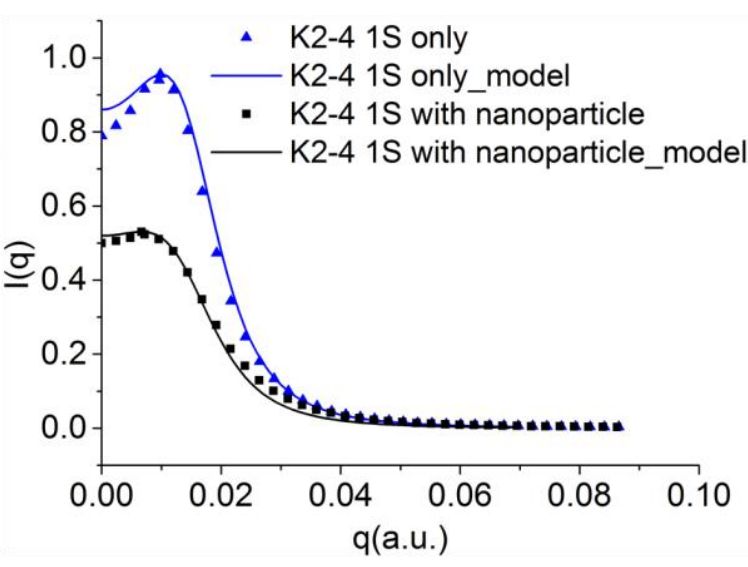

(b)

Figure 8. SAXS and Strey model simulation for octane-water-surfactant microemulsion both with and without $\mathrm{C}_{16}$-ssPVA-OCB (0.1 wt \%) nanoparticle added: (a) 2 wt \% K3-4 $1 \mathrm{~S}$ at salinity $12.2 \mathrm{wt} \%$ (b) $2 \mathrm{wt} \% \mathrm{~K} 2-41 \mathrm{~S}$ at salinity $15.4 \mathrm{wt} \% .2 .5 \mathrm{wt} \%$ sec-butyl alcohol is added in all cases. 
The model by Strey et al. was used to extract the domain size $d$ and correlation length $\xi$ from the data. ${ }^{35,36}$ As shown in Fig. 8, this model was able to capture the features of the low-angle scattering curve accurately and provide quantitative estimates for $d$ and $\xi$. For both surfactants, an approximately $15 \mathrm{~nm}$ increase in the domain size was measured in the presence of polymer-coated nanoparticles, as shown in Table 2. The polymer-coated nanoparticles are much larger than $15 \mathrm{~nm}$, and therefore this suggests that the polymer-coated nanoparticles occupy the oil-water interface with chains extended into both phases, as shown schematically in Figure 1. The interaction of the amphiphilic chains with the aqueous phase and the hydrophobic chains with the oil phase helps stabilize the nanoparticles at the interface, and a small but measureable increase in the domain size was observed.

Table 2. Domain size $d$ and correlation length $\xi$ for microemulsion phases formed by K2-4 $1 \mathrm{~S}$ and K3-4 1S surfactants both with and without $\mathrm{C}_{16}$-ssPVA-OCB nanoparticles present based on small-angle X-ray analysis of bicontinuous phases.

\begin{tabular}{ccc} 
Microemulsion stabilizer & $\mathrm{d} / \mathrm{nm}$ & $\xi / \mathrm{nm}$ \\
\hline K2-4 1S only & 49.1 & 121.8 \\
K3-4 1S only & 63.0 & 333.3 \\
\hline K2-4 1S with C 16 -SsPVA-OCB & 58.6 & 119.1 \\
K3-4 1S with C 16 -SsPVA-OCB & 74.3 & 162.1 \\
\hline
\end{tabular}

\section{CONCLUSIONS}

We synthesized a series of oxidized carbon black nanoparticles with covalently attached hydrophilic and hydrophobic chains and investigated the equilibrium phase behavior of these nanoparticles in the presence of oil and water. A mixture of hydrophilic PVA and hydrophobic chains $\mathrm{C}_{16}$ were observed to produce stable emulsions over a broad salinity range, and sulfation of 
the PVA chains significantly improved dispersion stability in aqueous media at elevated salinities. When mixed with surfactants that stabilized the formation of bicontinuous microemulsion phases, polymer-coated nanoparticles segregated strongly and preferentially to the middle phases. This reflects the interfacial activity of amphiphilic nanoparticle additives, and highly sulfated polymercoated nanoparticles $\mathrm{C}_{16}$-ssPVA-OCB were stable and surface active at elevated salinities, up to 15 wt $\% \mathrm{NaCl}$. A combination of IFT measurements and phase behavior studies showed that the nanoparticle additives had only a small impact on the IFT. Furthermore, SAXS measurements revealed a modest but measurable increase in the domain size on addition of the polymer-coated nanoparticles. These phase behavior studies clearly demonstrate that the use of an amphiphilic nanoparticle coating along with a charged, highly sulfated polymer results in polymer-coated nanoparticles that are stable at high salinities and migrate to microemulsion middle phases. Combined with other favorable properties of nanoparticle additives, we envision this work will lead to future developments of nanoparticle additives which can segregate to bicontinuous phases, propagate through porous media, modify interfacial properties, and enhanced the efficiency of enhanced oil recovery processes. The studies reported here can also be extended to other polymercoated nanoparticle systems, such as nanoparticles coated with poly(oligo(ethylene oxide) monomethyl ether methacrylate) which have shown to produce more substantial reductions in IFT at very low polymer-coated nanoparticle concentrations. We expect that the stability of the nanoparticle dispersion and the phase behavior will depend on the nanoparticle size and concentration, salinity, coverage of polymer on the surface, and compatibility of polymer chains with both oil and water. 


\section{AUTHOR INFORMATION}

\section{Corresponding Authors}

Rafael Verduzco, 6100 Main Street, MS-362, Houston, TX 77005, rafaelv@ rice.edu

George J. Hirasaki, 6100 Main Street, MS-362, Houston, TX 77005, gjh@ rice.edu

James M. Tour, 6100 Main Street, MS-60, Houston, TX 77005, tour@ rice.edu

Author Contributions

These authors contributed equally: Luqing Qi and Hadi ShamsiJazeyi

\section{ACKNOWLEDGEMENTS}

The authors of this work acknowledge the Advanced Energy Consortium (AEC) for financial support under project no. BEG08-007 and the Rice Univeristy Consortium for Processes in Porous Media.

\section{REFERENCES}

(1) Annual Energy Outlook, U.S. Energy Information Administration, http://www.eia.gov/analysis/projection-data.cfm

(2) Harnoy, G. N.; Gatt, S.; Barenholtz, Y. Enhanced oil recovery; Google Patents, 1989.

(3) Shah D.O.; Schechter R.S. Imporved Oil Recovery by Surfactant and Polymer Flooding; Academic Press: New York, NY, 1977

(4) Hirasaki, G.; Miller, C. A.; Puerto, M. Recent Advances in Surfactant EOR. SPE-115386-PA 2011.

(5) Zhang, H.; Nikolov, A.; Wasan, D. Enhanced Oil Recovery (EOR) Using Nanoparticle Dispersions: Underlying Mechanism and Imbibition Experiments. Energy Fuels 2014, 28 (5), 3002-3009.

(6) Binks, B. P.; Lumsdon, S. O. Pickering Emulsions Stabilized by Monodisperse Latex Particles: Effects of Particle Size. Langmuir 2001, 17 (15), 4540-4547.

(7) Binks, B. P. Particles as surfactants-similarities and differences. Curr. Opin. Colloid Interface Sci. 2002, 7 (1-2), 21-41.

(8) Binks, B. P.; Rodrigues, J. A. Enhanced Stabilization of Emulsions Due to SurfactantInduced Nanoparticle Flocculation. Langmuir 2007, 23 (14), 7436-7439.

(9) Binks, B. P.; Rodrigues, J. A.; Frith, W. J. Synergistic Interaction in Emulsions Stabilized by a Mixture of Silica Nanoparticles and Cationic Surfactant. Langmuir 2007, 23 (7), 36263636. 
(10) Yoon, K. Y.; Li, Z.; Neilson, B. M.; Lee, W.; Huh, C.; Bryant, S. L.; Bielawski, C. W.; Johnston, K. P. Effect of Adsorbed Amphiphilic Copolymers on the Interfacial Activity of Superparamagnetic Nanoclusters and the Emulsification of Oil in Water. Macromolecules 2012, 45 (12), 5157-5166.

(11) Bagaria, H. G.; Neilson, B. M.; Worthen, A. J.; Xue, Z.; Yoon, K. Y.; Cheng, V.; Lee, J. H.; Velagala, S.; Huh, C.; Bryant, S. L.; Bielawski, C. W.; Johnston, K. P. Adsorption of iron oxide nanoclusters stabilized with sulfonated copolymers on silica in concentrated $\mathrm{NaCl}$ and $\mathrm{CaCl} 2$ brine. J. Colloid Interface Sci. 2013, 398, 217-226.

(12) Xue, Z.; Foster, E.; Wang, Y.; Nayak, S.; Cheng, V.; Ngo, V. W.; Pennell, K. D.; Bielawski, C. W.; Johnston, K. P. Effect of Grafted Copolymer Composition on Iron Oxide Nanoparticle Stability and Transport in Porous Media at High Salinity. Energy Fuels 2014, 28 (6), 36553665.

(13) Foster, L. M.; Worthen, A. J.; Foster, E. L.; Dong, J.; Roach, C. M.; Metaxas, A. E.; Hardy, C. D.; Larsen, E. S.; Bollinger, J. A.; Truskett, T. M.; Bielawski, C. W.; Johnston, K. P. High Interfacial Activity of Polymers "Grafted through" Functionalized Iron Oxide Nanoparticle Clusters. Langmuir 2014, 30 (34), 10188-10196.

(14) Kim, D.; Krishnamoorti, R. Interfacial Activity of Poly[oligo(ethylene oxide)-monomethyl ether methacrylate]-Grafted Silica Nanoparticles. Ind. Eng. Chem. Res. 2015, 54 (14), 36483656.

(15) Villamizar, L. C.; Lohateeraparp, P.; Harwell, J. H.; Resasco, D. E.; Shiau, B. J. B. Interfacially Active SWNT/Silica Nanohybrid Used In Enhanced Oil Recovery. In SPE129901-MS; Society of Petroleum Engineers: SPE, 2010.

(16) Villamizar, L. C.; Lohateeraparp, P.; Harwell, J. H.; Resasco, D. E.; Shiau, B. J. Dispersion Stability and Transport of Nanohybrids through Porous Media. Transp. Porous Media 2013, 96 (1), 63-81.

(17) Cui, M.; Emrick, T.; Russell, T. P. Stabilizing Liquid Drops in Nonequilibrium Shapes by the Interfacial Jamming of Nanoparticles. Science 2013, 342 (6157), 460.

(18) Mirshahghassemi, S.; Lead, J. R. Oil Recovery from Water under Environmentally Relevant Conditions Using Magnetic Nanoparticles. Environ. Sci. Technol. 2015, 49 (19), 1172911736.

(19) Palchoudhury, S.; Lead, J. R. A Facile and Cost-Effective Method for Separation of OilWater Mixtures Using Polymer-Coated Iron Oxide Nanoparticles. Environ. Sci. Technol. 2014, 48 (24), 14558-14563.

(20) ShamsiJazeyi, H.; Miller, C. A.; Wong, M. S.; Tour, J. M.; Verduzco, R. Polymer-coated nanoparticles for enhanced oil recovery. J. Appl. Polym. Sci. 2014, 131 (15), 40576.

(21) Cheraghian, G.; Hendraningrat, L. A review on applications of nanotechnology in the enhanced oil recovery part A: effects of nanoparticles on interfacial tension. Int. Nano Lett. 2016, 6 (2), 129-138.

(22) Cheraghian, G.; Hendraningrat, L. A review on applications of nanotechnology in the enhanced oil recovery part B: effects of nanoparticles on flooding. Int. Nano Lett. 2016, 6 (1), 1-10.

(23) Holmberg, K.; Jönsson, B.; Kronberg, B.; Lindman, B. Surfactants and Polymers in Aqueous Solution; John Wiley and Sons: Chichester, West Sussex, England, 2002.

(24) Fanun, M. Microemulsions: Properties and Applications; Taylor and Francis: Boca Raton, FL, 2008.

(25) McClements, D. J. Nanoemulsions versus microemulsions: terminology, differences, and 
similarities. Soft Matter 2012, 8 (6), 1719-1729.

(26) Liu, S.; Miller, C. A.; Li, R. F.; Hirasaki, G. Alkaline/Surfactant/Polymer Processes: Wide Range of Conditions for Good Recovery. SPE-113936-PA 2010.

(27) Jiang, Z. GIXSGUI is available for download: http://www.aps.anl.gov/Sectors/Sector8/Operations/GIXSGUI.html.

(28) Cui, Z.-G.; Cui, Y.-Z.; Cui, C.-F.; Chen, Z.; Binks, B. P. Aqueous Foams Stabilized by in Situ Surface Activation of CaCO3 Nanoparticles via Adsorption of Anionic Surfactant. Langmuir 2010, 26 (15), 12567-12574.

(29) Limage, S.; Krägel, J.; Schmitt, M.; Dominici, C.; Miller, R.; Antoni, M. Rheology and Structure Formation in Diluted Mixed Particle-Surfactant Systems. Langmuir 2010, 26 (22), 16754-16761.

(30) Worthen, A. J.; Bryant, S. L.; Huh, C.; Johnston, K. P. Carbon dioxide-in-water foams stabilized with nanoparticles and surfactant acting in synergy. AIChE J. 2013, 59 (9), 34903501 .

(31) Binks, B. P.; Kirkland, M.; Rodrigues, J. A. Origin of stabilisation of aqueous foams in nanoparticle-surfactant mixtures. Soft Matter 2008, 4 (12), 2373-2382.

(32) Wolf, L.; Hoffmann, H.; Talmon, Y.; Teshigawara, T.; Watanabe, K. Cryo-TEM imaging of a novel microemulsion system of silicone oil with an anionic/nonionic surfactant mixture. Soft Matter 2010, 6 (21), 5367-5374.

(33) Huh, C. Interfacial tensions and solubilizing ability of a microemulsion phase that coexists with oil and brine. J. Colloid Interface Sci. 1979, 71 (2), 408-426.

(34) Puerto, M. C.; Gale, W. W. Estimation of Optimal Salinity and Solubilization Parameters for Alkylorthoxylene Sulfonate Mixtures. SPE-5814-PA 1977.

(35) Teubner, M.; Strey, R. Origin of the scattering peak in microemulsions. J. Chem. Phys. 1987, 87 (5), 3195-3200.

(36) Strey, R. Microemulsion microstructure and interfacial curvature. Colloid Polym. Sci. 1994, 272 (8), 1005-1019.

\section{Table of Contents Artwork}

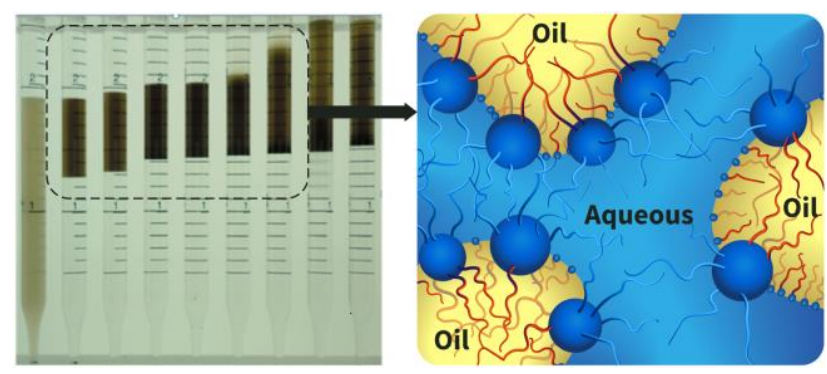

